Purpose To demonstrate the necessity for aseptic technique and conditions and preparation by the pharmacy.

Materials and Methods For a period of six months 15 patients were prescribed Bergman's solution $500 \mathrm{ml}$ to which was added $5 \mathrm{ml}$ pentoxiphylin and $12 \mathrm{ml}$ lidocaine $2 \%$ (50 ml vials divided between 4 patients) in the orthopaedic department. This infusion was prepared in the nursing room, by the nurses without suitable aseptic conditions. For the next six months pharmacists prepared this infusion in the hospital pharmacy aseptic facility. 17 patients in the orthopaedic department got this solution.

Results The nurses used each $50 \mathrm{ml}$ vial of lidocaine for several patients until the vial was used. The vial was saved for use the following day after initial entry. Within days of application 8 patients required antibiotics and prolonged hospitalisation. Microbiological tests showed MRSA infection. One of the nurses forgot to wash hands before preparing the infusion for 3 patients, one used the same needle for both drugs for 4 patients, and one accidentally touched the needle in 1 patient. In the next six months the hospital pharmacy prepared 17 infusions for 17 patients in the aseptic facility. All patients finished their treatment in very good condition without any complications.

Conclusions Nurses' rooms and training are unsuitable for reusing single dose vials for several patients. Subdividing must follow highly controlled environmental conditions, with training and qualifications of personnel and procedures for reuse, which are met by the hospital pharmacy and pharmacists in our hospital.

No conflict of interest.

\section{GRP-161 RISK ASSESSMENT FORMS FOR PHARMACY PREPARATION}

doi:10.1136/ejhpharm-2013-000276.161

Y Bouwman. Royal Dutch Pharmacists Association, Scientific Institute for Dutch Pharmacists, Den Haag, The Netherlands

Background Pharmacists are allowed to prepare medicines for the needs of patients. They have to balance the benefits and risks of the clinical and pharmaceutical qualities. In other words they have to perform a risk assessment for extemporaneous preparation as well as for stock preparation.

To perform a risk assessment the pharmacist should be able to list the benefits and risks and needs a tool to balance them. Some approaches have been published, but they don't deal with all aspects in one view. We think there is a need for a risk assessment tool that is simple, transparent and conclusive and that deals with all relevant aspects.

Purpose To analyse the pharmaceutical process for decisive steps, levels of evidence and actors. To incorporate these aspects into a practicable form.

Materials and Methods 15 years of feedback from community and hospital pharmacists on former assessment forms, discussions with authorities, 40 years searching for sound reasons for pharmacy preparation, writing an opinion on the Resolution on pharmacy preparation of the Council of Europe, have been used as an input for creating a new form that emphasises the benefit and risk balance.

Results Two forms were developed for the pharmacist: for extemporaneous and for stock preparation. They use the same type of benefit and risk aspects but extemporaneous preparation affects an assignable patient and the request is from an assignable physician. Often two pharmacists are involved, the attending pharmacist and the preparatory pharmacist. All four carry responsibility but the preparatory pharmacist has to decide whether to fulfil the request or not. For stock preparations the preparatory pharmacist will put together the information about benefits and risks. The physician, patient and attending pharmacist have to balance them. Stock preparation requires numerous items per batch and serves a number of patients. This requires a higher level of evidence about the clinical value and a higher quality of design.

Conclusions Forms were developed for the risk assessment of extemporaneous and stock preparations. They show decisions and provide transparency, pointing at responsibility and accountability. Practical experience will provide more information about the roles of pharmacist(s), physician and patient.

No conflict of interest.

\section{GRP-162 RISK CATEGORIZATION OF STANDARDISED CONTINUOUS INJECTION/-INFUSION SOLUTIONS AT THE UNIVERSITY MEDICAL CENTER MAINZ}

doi:10.1136/ejhpharm-2013-000276.162

B Zeiter, S Bellers, I Kraemer. University Medical Center, Pharmacy Department, Mainz, Germany

Background The prescription, preparation and use of parenteral solutions are complex processes composed of many steps, during which mistakes can occur. However, by means of the National Patient Safety Alert 20 (NPSA 20), a risk evaluation of continuous injection/-infusion solutions can be performed.

Purpose To evaluate the risks associated with the intravenous drug treatment of intensive care unit patients at the University Medical Center Mainz. We planned to use the results to identify high-risk products and implement measures to reduce potential risks.

Materials and Methods The NPSA 20 defines eight different risk factors for the evaluation of overall risk. The risk evaluation was conducted for 78 continuous injection/-infusion solutions used in intensive care unit patients. These parenteral solutions are used in standardised concentrations; 16 of them were prepared as ready-touse products in the hospital pharmacy. The potential risks of these 16 preparations were compared with the risks of those not prepared centrally in the hospital pharmacy department.

Results The risk evaluation of the 78 continuous injection/infusion solutions revealed that most of the standardised 78 solutions were moderate-risk products $(68 \%)$. Other solutions were classified as low-risk products (26\%). Only $6 \%$ of the solutions were high-risk products. The favourable results of the risk analysis can be explained by the hospital-wide use of standardised concentrations. Doses are adjusted by using the infusion rate. For a number of products $(12 \%)$ the risk category was downgraded from moderate to low, since ready-to-use products were prepared in the hospital pharmacy department.

Conclusions Out of 78 drug products administered as continuous injection/-infusion solutions to intensive care unit patients only $6 \%$ were categorised as high-risk. This favourable result is explained the use of standardised concentrations and preparation of ready-to-use products in the pharmacy department.

No conflict of interest.

\section{GRP-163 RISK MANAGEMENT MEASURES TO PREVENT PHYSICAL- CHEMICAL INCOMPATIBILITIES DURING CONTINUOUS IV INFUSION}

doi:10.1136/ejhpharm-2013-000276.163

${ }^{1} \mathrm{C}$ Linguadoca, 'S Parazza, 'E Musi, 'A Zanardi, ' $\mathrm{F}$ Caliumi, ${ }^{2} \mathrm{MV}$ Antonini, ${ }^{2} \mathrm{ML}$ Caspani. ${ }^{1}$ Azienda Ospedaliero-Universitaria Parma, Servizio di Farmacia e Governo Clinico del Farmaco, Parma, Italy; ${ }^{2}$ Azienda Ospedaliero-Universitaria Parma, I Servizio di Anestesia e Rianimazione, Parma, Italy

Background Patients in critical care (ICU) settings usually require multiple medicines administered as continuous IV infusions. As a reliable IV access is often unavailable, simultaneous administration through the same line is performed using a Y-site connector 